\title{
Technical Supervision Key Points of Smart Substation Secondary Professional Process
}

\author{
Yan Xiaohong \\ Shanxi Jincheng Power Supply Comapany \\ Jincheng, China \\ e-mail:yanxiaohong@sx.sgcc.com.cn
}

\section{Cheng Gang}

Shanxi Jincheng Power Supply Comapany

Jincheng, China

e-mail: 15934179895@163.com

\author{
Wang Guogang * \\ Beijing Guodian Futong Science and \\ Technology Development Co., Ltd \\ Beijing, China \\ e-mail: ssnabi@126.com
}

\author{
Han Jinfeng \\ Shanxi Jincheng Power Supply Comapany \\ Jincheng, China \\ e-mail: hanjinfeng@sx.sgcc.com.cn
}

\author{
Ma An \\ Shanxi Jincheng Power Supply Comapany \\ Jincheng, China \\ e-mail:maan@sx.sgcc.com.cn
}

\begin{abstract}
The smart substation construction is developing rapidly, and operation maintenance unit must be faced with the new problems which are brought about by the new technology. Based on more intelligent substation debugging, acceptance and operation experience, the main technical supervision points of the need to pay attention to and the detection method are enumerated from the stages of the design, plant secondary equipment alignment phase, site commissioning phase and the operation and maintenance of the intelligent substation secondary system in this article. For intelligent station secondary system design, construction, acceptance and operation, it must be constantly in practice to analyze the characteristics of the new technology, new equipment, combined with operating experience of conventional ensemble station. It should be taken reasonable personnel organization and processes to ensure safe and reliable operation of the intelligent station.
\end{abstract}

Keywords-smart substation; secondary system; techological supervision;

\section{INTRODUCTION}

Smart substation construction is an important part of the construction of strong smart grid work. With the rapid advance of intelligent substation construction and the rapid development of new technology, new applications, substation secondary equipment operation maintenance personnel will face a series of difficulties, such as, the intelligent substation design and construction process standardization, debugging and acceptance without elaboration standard, operation maintenance without experience. It is necessary to strengthen the whole process of intelligent substation construction and operational management.

\section{TECHNICAL SUPERVISION POINTS}

\section{A. Design Phase}

\section{1) Basic priciples}

The substation secondary substation design should ensure long-term safe and reliable operation after putting into operation.

The construction of equipment should consider the convenience of maintenance and overhaul work and convenient arrangement and simple logic of secondary safety measures.

Late design should be proactive to ensure that the substation expansion access work easy and the secondary system have compatibility.

\section{2) Technical supervisin points}

a) Primary equipment connection mode is simple; CT (Current Transformer) and PT (Pressure Transformer) quantity meet secondary professional requirements.

The logic and configure of secondary equipment is directly related to the link-wire way and quantity of primary equipment. The special link-wire way and an insufficient number are likely to lead to relay configuration difficulties, logic complexity, scope of protection failure and other problems. For example, $110 \mathrm{kV}$ expansion of the bridge connection, if secondary bus has no PT, will lead to voltage parallel logic complexity, overhaul difficulty of security measures.

b) Secondary equipment has reasonable configuration, perfect function, complete interface number.

The dual implementation should be protected. For example, the merging units and intelligent terminals with related to $110 \mathrm{kV}$ main transformer protection double configuration should have double configurations. Bus 
merging unit should have double configuration or main and backup configuration. The larger the combined unit optical port power is, the higher the failure rate is. The spare port should be left and configured completely. In addition, the spare port should be plugged and played if the next technology allows.

c) Careful with the secondary equipment in situ, optimize the environment of secondary equipment operation.

Protection equipment should be independent decentralized and locally installed. Installed operating environment should meet the relevant technical standards [1].

d) The installation position of GIS control cabinet and the intelligent control cabinet should be reasonable to avoid the operation maintenance personnel to enter wrong interval.

\section{e) Networking inspection}

The SV network and GOOSE network of the process layer and the MMS network of the station control layer of the $110 \mathrm{kV}$ and above voltage level should be completely independent. When protection devices connect to different networks, independent data interface controller should be used[2]. It has something wrong with the secondary curret waveform in Fig.1



Figure 1. Abnormity of the secondary current waveform

f) The secondary equipment access permission check

It is necessary to select well-known manufacturers of mature products and inspect equipment network license strictly. At present, the State Grid Corporation only announced the test report of merger unit and intelligent terminal of $220 \mathrm{kV}$ and above. The test report of $110 \mathrm{kV}$ and below is not issued yet, which still need to pay close attention [1, 3].

g) It needs to account the capacity of accumulator system strictly and determine the importance of all kinds of direct current $(D C)$ system load level to guarantee the exchange of relay protection, monitoring device, communication devices running time secondary access permission check

Integrated alternating current (AC)-DC power supplies are widely used in smart substation currently. In partial design, the auxiliary systems equipment such as fire, security video, the site video lighting equipment has access to UPS (Uninterrupted Power Supply). In case of AC power failure, the battery system is overloaded, especially in battery under aging, may cause a safety hazard.

\section{h) Information point table standardization}

It requirements that point table format information and content are in line with the National Grid Company "Typical Substation Information Table" requirements to avoid the wrong item and omission of information.

\section{i) Printer functional requirements}

To meet the commissioning, operation and maintenance need, it should be required to achieve reliable junction of network printer and protection devices. The print content and format are in accordance with the device printer. The protector should have printing interface and data lines. The fault wave record device should have an independent printer.

\section{B. Plant Secondary Equipment Alignment Phase}

\section{1) Basic principles}

In the factory the alignment should be geared to the needs of the particular system engineering, focusing on the SCD file configuration, intelligent communication, virtual terminal device configuration, etc., to ensure that each inquiry and interoperability between intelligent device, to create conditions for smoothly the scene debugging [4].

2) Technical supervisin points

a) Check the correctness of the relevant technical documents and their implementation

The documents include the contract and technical agreement, the design drawings and design change documents, technical liaison meeting minutes, the list of equipment and system configuration list, the equipment and system configuration of technical data and inspection report, the total station configuration description file (SCD)version information and the factory debugging outline.

\section{b) Total station scheduling number and name}

The design data should be provided to related operation department before alignment by design units [5], so that number and total station scheduling and name are defined as soon as possible. SCD is the core content of the secondary system alignment work, which contains information dispatch number, name, etc. In alignment process, if the total scheduling number with each interval branch name cannot be determined and provided to the integrator in time, it will affect integrator of SCD configuration and the configuration file is not completed at once.

\section{c) Check profile}

The intelligent electronic device (IED) configuration check, SCD virtual terminal associated configuration check, total station switch configuration check, and the configuration inspection of Stand accused layer equipment [6].

\section{d) Check file statute syntax}

The IED file statute syntax of the secondary intelligent device and SCD file statute syntax should be checked.

\section{e) Information security test}

The background of network, which is made by integrator, should have access to information security test 
before the factory alignment, especially the firewall to ensure information security.

\section{f) A digital dynamic model test}

If the conditions allow, the total station system should have dynamic model test to ensure that total station equipment in various operation conditions are working properly and to put forward measures for possible safety concerns.

\section{Site Commissioning Phase}

\section{1) Basic principles}

The commissioning should manage and control various work on the basis of the preparation of the debugging condition and basic work, and in accordance with the "debugging preparation, monomer debugging, subsystem debugging, and total station synchronous sampling verification test" level to ensure the quality of commissioning and debugging.

The version of the program files and configuration files should be strengthened in the process of debugging. The intelligent device parameter configuration should also be controlled strictly. Installation and debugging unit shall be responsible for the management to avoid equipment manufacturer do changes and modification without authorization.

In debugging process, if the primary equipment already charged operation, some effective measures should be taken to isolate the corresponding system and the system configuration changes should be incorporated into the site operation safety management.

Network analysis, fault wave record and other network data subscription equipment in the project configuration, should adopt the direct method to import engineering SCD file configuration to ensure the uniqueness of the SCD file total station.

Intelligent device network adaptor, port parameter settings should be consistent with the engineering design. It should be checked about the effectiveness, stability and uniqueness of the information transmission of process layer network [7].

In the secondary cable connection parts between intelligent component and the primary equipment, in accordance with requirements of the traditional implementation, the cable should be deployed in a special cable groove to avoid mixed with cable arrangement.

\section{2) Technical supervisin points}

\section{a) Debugging preparation}

Equipment installation and the secondary system network are complete. The environmental conditions meet the requirements of commissioning on site.

The relevant files, which include approval documents, design drawings, equipment manuals, SCD file, and intelligent device model, should be complete and correct. The virtual terminal and related description are in accordance with design and engineering practice.

The AC/DC system and grounding system meet the requirements of debugging.

Commissioning instrumentation is complete. The instrument must pass inspection, and during the validity period of inspection.

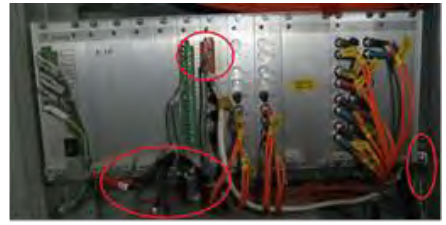

Figure 2. Anti-interference measures

\section{b) Monomer debugging}

Monomer debugging includes interval layer, process of protection device, merging unit, intelligent terminal, so the recording devices such as switches, mesh points within the functional verification and screen monomer related secondary loop check and input/output signals. The main supervision and technical key points should include SV correctness packet inspection, GOOSE messages correctness checks, check protective device maintenance mechanism, combined wisdom devices and check anti-interference measures and outdoor intelligent tank inspection in Fig.2.

\section{c) Subsystem commissioning work}

The debugging work mainly includes the stand accused of layer, spacer layer, process of secondary equipment protection, validity tests the linkage of the measurement and control, monitoring, and other functions. The main supervision and technical key points should include Detection process layer network straight mining straight jump the validity of the information transmission, stability and uniqueness, SV, GOOSE link abnormal action of protection device check, Time Synchronization System check and the management and check of the soft background monitoring system of relay protection linking piece and the inspection of the attenuation, process and number of fiber optical cable end in Fig.3. In Fig.4 the fiber optical cable has emerged obvious heating.

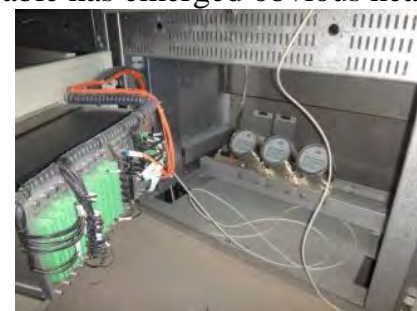

Figure 3. The fiber optical cable

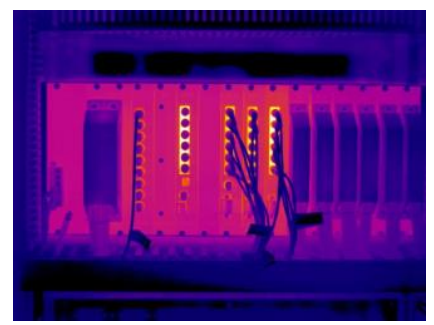

Figure 4. The heating phenomenon of fiber

Samples values communication configuration, virtual terminal connections shall be consistent with the SCD file; SV into linking piece should be consistent with the data input of SV, inconsistent device shall be submitted to the sampling faults, locking related protection at the same time. 
GOOSE virtual terminal open enter and leave should be consistent with the SCD file; GOOSE virtual terminal output in SCD file sending data collection of DOI have clear loop in the item description should be defined; GOOSE chain scission, inconsistent condition, the device should be given corresponding warning message and the message should be reported to station level at the same time.

d) Total station synchronous sampling confirmatory test

The most important difference between the conventional ensemble substation and smart substation is that the collection way of protection voltage and power flow is different. The conventional analog acquisition does not have delay problems, but the digital quantity acquisition switch delay. If the current and voltage which are obtained by protection device are out of sync, it will lead to serious accidents. Henan $500 \mathrm{KV}$ substation area protection disoperation accident is due to this reason.

Use qualified protection tester to check different merger involving the same protection unit to analog at the same time, see, wave record sampling of protection device, which to a certain extent, inspection device of synchronicity. It was detected on both sides of main transformer differential protection due to merge unit electricity flow out of sync lead to the difference between the abnormal flows.

\section{Operation and Maintance Phase}

\section{1) Basic principles}

It should be clear about the difference between the intelligence station and the conventional ensemble from standing. The content about the "safety management, operation management, equipment management, data management" of the secondary system should be improved to enhance the operation reliability of the substation secondary system [8].

2) Technical supervisin points

Prepare the clamp instructions, clear the logical relationship between soft clamp and hard clamp.

Operation rules should be increased in the station control layer, spacer layer, process the network structure and the transmission of a message form, the abnormal situations of network processing scheme, clear public switch network equipment such as troubleshooting when the scope of protection should be discontinued.

The intelligent substation maintenance shall be equipped with the special laptop. Pay attention to network security.
The final version SCD file, the MAC address of each smart device, APPID and other electronic document should be on file and backed up properly to prevent the loss due to the computer fault.

The maintenance unit should study the intelligent station structure characteristics seriously, and make secondary equipment safety measures in advance.

Strengthen the content training for operation maintenance personnel, which is related to intelligence station, and actively communicate with other smart stations and learn operating maintenance experience.

Key jobs should be moved forward. In combination with intelligent substation secondary integrated management platform construction in Shanxi, the smart station equipment type selection, design and alignment should be kept relevant records.

\section{CONCLUSION}

For intelligent station secondary system design, construction, acceptance and operation, it must be constantly in practice to analyze the characteristics of the new technology, new equipment, combined with operating experience of conventional ensemble station. It should be taken reasonable personnel organization and processes to ensure safe and reliable operation of the intelligent station.

\section{ACKNOWLEDGMENT}

The financial support of this work extended by the Science and Technology Project of State Grid Shanxi Electric Power Company (Grant 5205E01351DG) and the National Natural Science Foundation of China (Grant 51007083) are gratefully acknowledged.

\section{REFERENCES}

[1] Q/GDW441-2010. Techical Specificaion of Protection for Smart Substation.

[2] Test Specification of Relay Protection for Smart Substation

[3] Inspection Speciication of Relay Protection for Smart Substation.

[4] Q/GDW393-2009. Design Code for Smart Substation.

[5] Q/GDW426-2010. Techical Specificaion of Merging Unit for Smart Substation.

[6] Q/GDW428-2010. Techical Specificaion of Intelligent Terminal for Smart Substation.

[7] Q/GDW429-2010. Techical Specificaion of Network Switch for Smart Substation.

[8] Q/GDW383-2009. Technical Guidelines for Smart Substation. 